\title{
Development of new chitosan/carrageenan nanoparticles for drug delivery applications
}

\author{
Ana Grenha, Manuela E. Gomes, Márcia Rodrigues, Vítor E. Santo, João F. Mano, \\ Nuno M. Neves, Rui L. Reis \\ Department of Polymer Engineering, University of Minho, 3Bs Research Group, Biomaterials, Biodegradables and \\ Biomimetics, Headquarters of the European Institute of Excellence on Tissue Engineering and Regenerative Medicine, \\ IBB Institute for Biotechnology and Bioengineering, PT Government Associated Laboratory, Portugal
}

Received 6 September 2008; revised 17 December 2008; accepted 29 January 2009

Published online 25 March 2009 in Wiley InterScience (www.interscience.wiley.com). DOI: 10.1002/jbm.a.32466

\begin{abstract}
The use of polymeric nanoparticles, especially those composed of natural polymers, has become a very interesting approach in drug delivery, mainly because of the advantages offered by their small dimensions. The aim of this work was to develop a novel formulation of nanoparticles comprised of two natural marine-derived polymers, namely chitosan and carrageenan, and to evaluate their potential for the association and controlled release of macromolecules. Nanoparticles were obtained in a hydrophilic environment, under very mild conditions, avoiding the use of organic solvents or other aggressive technologies for their preparation. The developed nanocarriers presented sizes within $350-650 \mathrm{~nm}$ and positive zeta potentials of $50-60 \mathrm{mV}$. Polymeric interactions between nanoparticles' components were evaluated by Fourier transform infrared spectroscopy. Using ovalbumin as model protein,
\end{abstract}

nanoparticles evidenced loading capacity varying from $4 \%$ to $17 \%$ and demonstrated excellent capacity to provide a controlled release for up to 3 weeks. Furthermore, nanoparticles have demonstrated to exhibit a noncytotoxic behavior in biological in vitro tests performed using L929 fibroblasts, which is critical regarding the biocompatibility of those carriers. In summary, the developed chitosan-carrageenan nanoparticles have shown promising properties to be used as carriers of therapeutic macromolecules, with potential application not only strictly in drug delivery, but also in broader areas, such as tissue engineering and regenerative medicine. (c) 2009 Wiley Periodicals, Inc. J Biomed Mater Res 92A: 1265-1272, 2010

Key words: carrageenan; chitosan; controlled release; ionic interaction; nanoparticles

\section{INTRODUCTION}

Natural polymers have been gaining increasing interest in the biomedical field and particularly in the area of drug delivery, either intrinsically or in the context of broader fields, such as tissue engineering. Amongst these polymers, polysaccharides found a wide variety of applications, given their participation in important functions of the organism, like membrane structure and intracellular communication, and also because of their flexibility, which allows polysaccharides to form both linear and branched polymers. ${ }^{1}$

Correspondence to: A. Grenha; e-mail: amgrenha@ualg.pt Contract grant sponsor: Portuguese Foundation for Science and Technology (FCT) (POCTI/FEDER programmes)

Contract grant sponsor: European Union STREP Project HIPPOCRATES; contract grant number: NMP3-CT-2003505758

Contract grant sponsor: European NoE EXPERTISSUES; contract grant number: NMP3-CT-2004-500283

(C) 2009 Wiley Periodicals, Inc.
Chitosan is a polymer of natural origin, which is composed of repeating units of $\mathrm{N}$-acetylglucosamine and D-glucosamine, being obtained from the deacetylation of chitin, the main component of the exoskeleton of crustaceans. ${ }^{2-4}$ This polysaccharide possesses structural characteristics similar to those displayed by glycosaminoglycans (GAGs), which are an important component of connective tissues and, owing to that feature, chitosan has been investigated for a range of biomedical applications, such as wound healing, tissue engineering, dentistry, and orthopedics. ${ }^{4}$ This polymer presents well-documented favorable biological properties such as biocompatibility, biodegradability, and low toxicity, ${ }^{5,6}$ and it also displays mucoadhesive properties, ${ }^{7}$ rendering this molecule very attractive for drug delivery applications.

Carrageenan is also a polysaccharide, extracted from red seaweed, which is composed of galactose and anhydrogalactose units, linked by glycosidic unions. Depending on the method and the algae from which carrageenan is extracted, three main types of carrageenans can be obtained, kappa (к), 
iota ( 1 ), and lambda $(\lambda)$, which mainly differ structurally in the degree of substitution of the sulfate group. ${ }^{8}$ Given the ionic nature of the polymer, its gelation is strongly influenced by the presence of electrolytes and, from these three types, only k- and 1-carrageenans evidence gel-forming ability, the k-carrageenan gels being more firm than those obtained with t-carrageenan, which are more elastic and soft. ${ }^{9}$

When two polyelectrolytes of opposite charge are combined, the formation of a physical hydrogel might take place, which is called ionotropic hydrogel. This hydrogel is held together by molecular entanglements, ionic forces, H-bonding, or hydrophobic forces. ${ }^{10}$ All these interactions are reversible and can be disrupted by changes in physical conditions such as ionic strength, $\mathrm{pH}$, and temperature. ${ }^{8}$ Depending on the concentrations of the polyelectrolytes, different gel-structures can be produced, such as beads, microparticles, or nanoparticles. Polyelectrolyte complexes of chitosan and carrageenan in the form of beads and tablets were previously reported to efficiently deliver sodium diclofenac and diltiazem hydrochloride, respectively. ${ }^{11,12}$ The formulation of nanoparticles as drug delivery systems would be very advantageous. In fact, because of their small dimensions, surface-to-volume ratio and surface functionality are enormously increased, rendering nanoparticles very promising vehicles for drug delivery. ${ }^{13,14}$

In this work, we report the preparation and characterization of protein-loaded nanoparticles obtained by ionic complexation of chitosan and carrageenan, as a valuable drug delivery system. For this purpose, we chose ovalbumin as model protein to be associated to the nanoparticles. Chitosan and carrageenan are naturally occurring polymers and are approved for applications in the food industry. Protein-loaded chitosan/ carrageenan nanoparticles were obtained by means of ionic complexation between the polyelectrolytes; their physicochemical and morphological properties were characterized and their ability to deliver the encapsulated macromolecule was evaluated in vitro. Moreover, the biocompatibility of the nanoparticles was assessed using a rat fibroblasts cell line.

\section{MATERIALS AND METHODS}

\section{Materials}

Chitosan (CS) (low molecular weigh, deacetylation degree $=75-85 \%$ ), albumin from chicken egg white (ovalbumin) grade $\mathrm{V}$, tungstophosphoric acid, phosphate buffered saline tablets (PBS) pH 7.4, Dulbecco's modified Eagle's medium low glucose, Dulbecco's modified Eagle's medium without phenol red and sodium bicarbonate were purchased from Sigma Chemicals (Germany). K-carrageenan (CRG) was obtained from Fluka Biochemica (Ger- many) and gycerol from Riedel-del-Haën (Germany). Fetal bovine serum (FBS) was from Biochrom AG, (Germany) and antibiotic-antimycotic (A/B) solution from Gibco (Spain). MTS was purchased from Promega (UK). Ultrapure water (TKA, Germany) was used throughout. All other chemicals were reagent grade.

\section{Determination of chitosan average viscosity molecular weight}

Prior to the determination of chitosan average viscosity molecular weight, chitosan was purified by a recrystalization procedure. Briefly, chitosan was dissolved in $1 \%(\mathrm{v} / \mathrm{v})$ acetic acid in order to achieve a concentration of $1 \mathrm{mg} /$ $\mathrm{mL}$. On complete dissolution, chitosan was filtered and neutralized with $\mathrm{NaOH}$ until $\mathrm{pH}$. The obtained precipitate was afterwards washed with water and freeze-dried for 4 days (Telstar Cryodos E79LH, IMA-Telstar, Spain).

To determine chitosan molecular weight, the purified chitosan was dissolved in $1 \%(\mathrm{v} / \mathrm{v})$ acetic acid to obtain different solutions in a range of concentrations, which were assayed for their flow time in a viscosimeter (MGW Werk Lauda, model D6970, Germany). Afterwards, the Mark-Houwink Eq. (1) was applied to determine the molecular weight (MW):

$$
[\eta]=k(\mathrm{MW})^{a}
$$

where $[\eta]$ is the intrinsic viscosity, determined by the viscosimeter, $k=3.5 \times 10^{-4}$ and $a=0.76$. $^{15}$

\section{Preparation of chitosan/carrageenan nanoparticles}

Chitosan/carrageenan (CS/CRG) nanoparticles were prepared by ionic complexation, ${ }^{16}$ by means of an electrostatic interaction of CS with CRG anions, in which the positively charged amino groups of CS interact with the negatively charged sulfate groups of CRG. Briefly, CS was dissolved in $1 \%(\mathrm{v} / \mathrm{v})$ acetic acid and CRG was dissolved in purified water at $60^{\circ} \mathrm{C}$, to obtain solutions of $1 \mathrm{mg} / \mathrm{mL}$ $(\mathrm{w} / \mathrm{v})$ and 0.42 to $0.71 \mathrm{mg} / \mathrm{mL}(\mathrm{w} / \mathrm{v})$, respectively, to reach final theoretical CS/CRG ratios of $3.5 / 1,4 / 1,5 / 1$, and $6 / 1(\mathrm{w} / \mathrm{w})$. The nanoparticles were spontaneously formed on incorporation of $1.2 \mathrm{~mL}$ of the different concentration CRG solutions into $3 \mathrm{~mL}$ of the CS solution, under magnetic stirring at room temperature. To prepare the protein-loaded CS/CRG nanoparticles, ovalbumin was dissolved in water (solution $\mathrm{pH}=6.2$ ) and further incorporated in the CRG solution afterwards $(\mathrm{pH}=7.6)$. The concentration of ovalbumin in the CRG solution was such that allowed preparing nanoparticles with 10,20 , and $30 \%$ (w/ w) of protein respective to the CS content. Nanoparticles were concentrated by centrifugation at $11,500 \mathrm{~g}$ on a $10-\mu \mathrm{L}$ glycerol layer, for $30 \mathrm{~min}$ (Biocen, Orto Alresa, Spain), glycerol being used to aid the subsequent resuspension. The supernatants were discarded and nanoparticles were resuspended in $100 \mu \mathrm{L}$ of purified water.

\section{Determination of nanoparticles production yield}

The nanoparticles production yield was determined by gravimetry. For this purpose, fixed volumes of nanoparticle suspensions were centrifuged $(11,500 \mathrm{~g}, 30 \mathrm{~min})$ and sedi- 
ments were freeze-dried over $24 \mathrm{~h}$, using a Telstar Freeze Dryer (Telstar Cryodos E79LH, IMA-Telstar, Spain) $(n=4)$.

The process yield was calculated as follows:

$$
\text { Process yield }(\%)=\frac{\text { Nanoparticles weight }}{\text { Total solids weight }} \times 100
$$

where nanoparticles weight is the sediment weight after freeze-drying and total solids weight is the total amount of solids added for nanoparticles formation (chitosan and carrageenan for unloaded nanoparticles and chitosan, carrageenan, and ovalbumin for protein-loaded nanoparticles).

\section{Characterization of nanoparticles}

\section{Morphology}

The morphological analysis of the nanoparticles developed in this study was performed by transmission electron microscopy (TEM) (CM 12 Philips, Eindhoven, Netherlands). Before TEM observation, samples were stained with $2 \%(\mathrm{w} / \mathrm{v})$ tungstophosphoric acid and placed on copper grids with Formvar ${ }^{\mathbb{B}}$ films.

Physicochemical properties

The size and zeta potential of the nanoparticles were measured by photon correlation spectroscopy and laser Doppler anemometry, respectively, using a Malvern Zetasizer $^{\circledR} 3000$ HS (Malvern Instruments, UK). For the particle size analysis, each sample was diluted to the appropriate concentration with water. Each analysis lasted $120 \mathrm{~s}$ and was performed at $25^{\circ} \mathrm{C}$ with a detection angle of $90^{\circ}$. For the determination of the electrophoretic mobility, samples were diluted with $\mathrm{KCl} 0.1 \mathrm{mM}$. Each formulation was analyzed in triplicate $(n=3)$.

Fourier transform infrared

The interactions between the different components of the nanoparticulate systems were analyzed by Fourier transform infrared (FTIR). Infra-red spectra of the specimen powders, namely chitosan, carrageenan, and chitosan/carrageenan nanoparticles, were recorded using a FTIR spectrophotometer (Shimadzu, IR Prestige-21, Japan). Prior to the assay, the samples were gently triturated with $\mathrm{KBr}$ (Riedel-del-Haën, Germany) and compressed into discs by pressing the powders. For each spectrum a 32-scan interferogram was collected in absorption with a $4 \mathrm{~cm}^{-1}$ resolution from the $4000-$ $400 \mathrm{~cm}^{-1}$ region at room temperature.

\section{Determination of the in vitro cytotoxicity of the chitosan/carrageenan nanoparticles}

L929 fibroblasts cell line

Mouse fibroblastic-like cell line L929, obtained from ECACC (UK), was grown in a humidified $5 \% \mathrm{CO}_{2} / 95 \%$ atmospheric air incubator, at $37^{\circ} \mathrm{C}$, using $75 \mathrm{~cm}^{2}$ flasks. The culture was maintained with Dulbecco's Modified Eagle's Medium low glucose (DMEM), supplemented with
$10 \%$ fetal bovine serum (FBS), 1\% antibiotic-antimycotic (A/B) solution, and sodium bicarbonate. Medium was changed every 3 days.

\section{MTS test}

The in vitro cytotoxicity of chitosan/carrageenan nanoparticles in the L929 cells was determined by the MTS (3(4,5-dimethylthiazol-2-yl)-5-(3-carboxymethoxyphenyl)-2(4-sulfophenyl)-2-tetrazolium) assay. Cells were seeded at a density of $4 \times 10^{3}$ cells/well in 96-well Costar flat bottom plates (Corning, USA), cultured with $200 \mu \mathrm{L}$ of the medium used to expand L929 cells, and allowed to grow at $37^{\circ} \mathrm{C}$ in a $5 \% \mathrm{CO}_{2}$ atmosphere for $24 \mathrm{~h}$, before cell viability assays. Before incubation with the test formulations, the culture medium was aspirated and the cells washed with PBS pH 7.4.

The chitosan/carrageenan nanoparticles (CS/CRG $=3.5$ / 1) were assayed for cytotoxicity at different concentrations $(0.1,1$, and $3 \mathrm{mg}$ nanoparticles $/ \mathrm{mL})$ for 1,3 , and 7 days in direct contact with L929 cells. Six replicates were considered per sample. All formulations were prepared as suspensions in prewarmed DMEM without phenol red containing $10 \%$ FBS and $1 \% \mathrm{~A} / \mathrm{B}$, immediately before their application to the cells. L929 cells relative viability (\%) was determined for each concentration of nanoparticles and compared with that of cells incubated only with cell culture medium without phenol red. Latex discs were used as a positive control of cell death. All tests were performed in triplicate.

Determination of protein loading capacity of nanoparticles

The nanoparticles association efficiency was determined upon their separation from the preparation medium containing the nonassociated protein by centrifugation $(11,500 \mathrm{~g}, 30 \mathrm{~min})$. The amount of free ovalbumin was determined in the supernatant by the MicroBCA protein assay (Pierce, USA), measuring the absorbances by spectrophotometry (Shimadzu UV-Visible Spectrophotometer UV-1603, Japan) at $562 \mathrm{~nm}$. A calibration curve was made using the supernatant of blank nanoparticles. Each sample was assayed in triplicate $(n=3)$. The nanoparticles' protein association efficiency and loading capacity were calculated from Eqs. (3) and (4) indicated below:

$$
\begin{aligned}
& \text { Association efficiency }(\%) \\
& =\frac{\text { Total amount of protein }- \text { Free amount of protein }}{\text { Total amount of protein }} \times 100 \\
& \text { Loading capacity }(\%) \\
& =\frac{\text { Total protein amount }- \text { Free protein amount }}{\text { Nanoparticles weight }} \times 100
\end{aligned}
$$

\section{In vitro release studies of ovalbumin from CS/CRG nanoparticles}

The release of ovalbumin was determined by incubating the nanoparticles $(\mathrm{CS} / \mathrm{CRG}=3.5: 1$ containing $30 \% \mathrm{w} / \mathrm{w}$ 


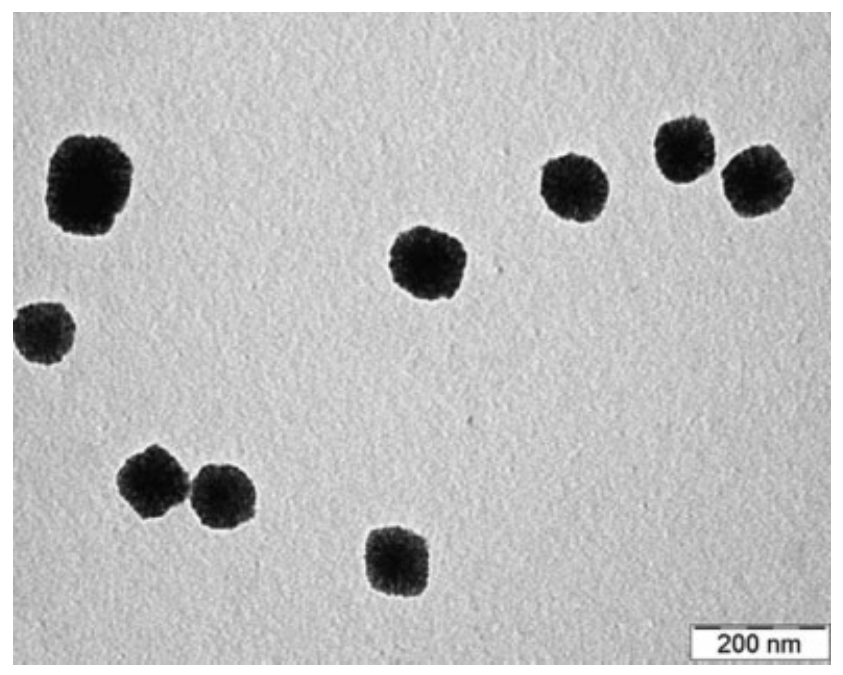

Figure 1. TEM microphotograph of chitosan/carrageenan $(3.5 / 1)$ nanoparticles.

ovalbumin based on chitosan) in $12 \mathrm{~mL}$ of $\mathrm{pH} 7.4$ phosphate buffer $(0.076 \mathrm{mg}$ nanoparticles $/ \mathrm{mL})$, with horizontal shaking, at $37^{\circ} \mathrm{C}$. Ovalbumin was in sink conditions (ovalbumin solubility at $\mathrm{pH} 7$ is $\sim 40 \mathrm{mg} / \mathrm{mL}$ ).

At appropriate time intervals (up to 3 weeks) individual samples were filtered $\left(0.22-\mu \mathrm{m}\right.$ filters Millex ${ }^{\circledR}-\mathrm{GV}$, low protein binding, Millipore, Portugal) and the amount of protein released evaluated in the supernatants by the MicroBCA protein assay (Pierce, USA) measuring the absorbances by spectrophotometry (Shimadzu UV-Visible Spectrophotometer UV-1603, Japan) at $562 \mathrm{~nm}(n=3)$.

\section{Statistical analysis}

The $t$-test and the one-way analysis of variance (ANOVA) with the pairwise multiple comparison procedures (Student-Newman-Kleus Method) were performed to compare two or multiple groups, respectively. All analyses were run using the SigmaStat statistical program (Version 3, SyStat, USA) and differences were considered to be significant at a level of $p<0.05$.

\section{RESULTS AND DISCUSSION}

\section{Preparation and characterization of chitosan/ carrageenan nanoparticles}

The average viscosity molecular weight of chitosan was determined to be $\sim 116 \mathrm{KDa}$, as obtained by the Mark-Houwink equation (data not shown).

Although the technique of ionic complexation was applied in other occasions to obtain chitosan nanoparticles by interaction with counter-anions, such as tripolyphosphate or dextran sulfate, ${ }^{16,17}$ the present work reports for the first time the production of chi- tosan nanoparticles by complexation with carrageenan, a natural polymer obtained from seaweed.

Chitosan/carrageenan (CS/CRG) nanoparticles were successfully obtained using a $1 \mathrm{mg} / \mathrm{mL}$ CS solution and CS/CRG mass ratios of 3.5/1, 4/1, 5/1, and $6 / 1$, by means of an electrostatic interaction between the positively charged chitosan amino groups and the oppositely charged sulfate groups of carrageenan. Ratios above 3.5/1 led to thorough precipitation, whilst mass ratios under $6 / 1$ did not lead to the formation of nanoparticles. This is attributed to the fact that when high CS/CRG mass ratios are employed (for instance, $2 / 1$ or $3 / 1$ ) the amount of carrageenan is too high compared with the chitosan amino groups available for neutralization, thus leading to precipitation. On the contrary, when the amount of carrageenan is too low (for example in a mass ratio of $7 / 1$ ), the amount of counter-anion is not sufficient to lead to the formation of nanoparticles. Figure 1 displays the TEM microphotograph of representative CS/CRG nanoparticles, which evidence a solid and compact structure, showing a tendency to exhibit a spherical-like shape.

As shown in Table I, the nanoparticles production yield was influenced by the CS/CRG ratio. More specifically, the incorporation of increasing amounts of CRG with respect to CS, led to a slight increase in the process yield, which is actually significantly higher for the formulation with CS/CRG mass ratio of 3.5/1 $(p<0.05)$, which achieved the maximum yield $(\sim 27 \%)$. This effect can be easily explained by the nanoparticles formation mechanism: as mentioned above, nanoparticles are formed as result of the interaction of the positively charged CS molecules with the counter-anion CRG and, therefore, the incorporation of higher amounts of CRG (lower CS/ CRG theoretical ratios) leads to the formation of a greater number of particles. ${ }^{18}$

As revealed by the photon correlation spectroscopy and laser Doppler anemometry analyses, unloaded CS/CRG nanoparticles displayed a particle size in the nanometric range, between $\sim 430 \mathrm{~nm}$ and $640 \mathrm{~nm}$, and a positive zeta potential within $+55 \mathrm{mV}$ and $+60 \mathrm{mV}$. The nanoparticles size increased progressively and significantly $(p<0.05)$ with the

TABLE I

Production Yields and Physicochemical Characteristics of Unloaded Chitosan/Carrageenan (CS/CRG) Nanoparticles (Mean \pm S.D.)

\begin{tabular}{cccc}
\hline CS/CRG & $\begin{array}{c}\text { Production } \\
\text { Yield }(\%)\end{array}$ & $\begin{array}{c}\text { Size } \\
(\mathrm{nm})\end{array}$ & $\begin{array}{c}\text { Zeta } \\
\text { Potential }(\mathrm{mV})\end{array}$ \\
\hline $3.5 / 1$ & $27 \pm 1$ & $634 \pm 54$ & $+55.9 \pm 1.0$ \\
$4 / 1$ & $21 \pm 4$ & $577 \pm 13$ & $+55.3 \pm 0.9$ \\
$5 / 1$ & $20 \pm 2$ & $500 \pm 11$ & $+60.2 \pm 0.3$ \\
$6 / 1$ & $18 \pm 4$ & $430 \pm 22$ & $+59.2 \pm 2.2$ \\
\hline
\end{tabular}


TABLE II

Production Yields and Physicochemical Characteristics of Ovalbumin-Loaded Chitosan/Carrageenan (CS/CRG) Nanoparticles (mean \pm S.D.)

\begin{tabular}{lcccc}
\hline & $\begin{array}{c}\text { Initial } \\
\text { Amount } \\
\text { of Protein } \\
(\%)^{\mathrm{a}}\end{array}$ & $\begin{array}{c}\text { Production } \\
\text { Yield } \\
(\%)\end{array}$ & $\begin{array}{c}\text { Size } \\
(\mathrm{nm})\end{array}$ & $\begin{array}{c}\text { Zeta } \\
\text { Potential } \\
(\mathrm{mV})\end{array}$ \\
\hline $3.5 / 1$ & 10 & $20 \pm 3$ & $446 \pm 14$ & $+49.5 \pm 3.4$ \\
$3.5 / 1$ & 20 & $21 \pm 3$ & $458 \pm 31$ & $+50.3 \pm 1.1$ \\
$3.5 / 1$ & 30 & $19 \pm 2$ & $402 \pm 28$ & $+49.4 \pm 1.6$ \\
$4 / 1$ & 10 & $20 \pm 3$ & $413 \pm 52$ & $+49.8 \pm 1.3$ \\
$4 / 1$ & 20 & $18 \pm 2$ & $346 \pm 46$ & $+52.9 \pm 3.3$ \\
$4 / 1$ & 30 & $18 \pm 4$ & $356 \pm 22$ & $+54.2 \pm 2.1$ \\
\hline
\end{tabular}

${ }^{\mathrm{a}}$ Relative to chitosan (w/w).

incorporation of increasing amounts of carrageenan $(\mathrm{CS} / \mathrm{CRG}=6 / 1$ to $3.5 / 1)$. This is quite logical, since carrageenan is a large polymer and, thus, higher amounts $(\mathrm{CS} / \mathrm{CRG}=3.5 / 1)$ led to the formation of larger particles. Higher amounts of CRG resulted in lower zeta potential values, the formulations 3.5/1 and $4 / 1$ presenting a lower surface charge $(p<0.05)$ as compared with formulations $5 / 1$ and $6 / 1$. This effect can be attributed to the higher neutralization of the CS positive amino groups by the negatively charged sulfate groups of CRG. ${ }^{19}$

The association of ovalbumin as model protein was investigated for nanoparticles with CS/TPP ratios of 3.5/1 and $4 / 1$, because those were the conditions showing higher production yields. The comparison of results presented in Tables I and II, corresponding to the unloaded and the ovalbumin-loaded nanoparticles, respectively, indicated that nanoparticles' size decreased significantly $(p<0.05)$ with the incorporation of ovalbumin in both formulations with either 10,20 , or $30 \%$ initial amount of ovalbumin. This decrease was from $634 \mathrm{~nm}$ (unloaded nanoparticles) to approximately $400-450 \mathrm{~nm}$ (ovalbumin-loaded) in the formulation CS/CRG $=3.5 / 1$ and from $577 \mathrm{~nm}$ (unloaded nanoparticles) to 350$400 \mathrm{~nm}$ (ovalbumin-loaded) in formulation 4/1. This reduction in size is possibly due to an effect of reticulation of the particles induced by the presence of the protein.

The incorporation of ovalbumin in the nanoparticles led, in some cases, to a statistically significant, although minor in value, decrease of zeta potential $(p<0.05)$, particularly for the formulation 3.5/1. Taking into account that ovalbumin is dissolved in water, the protein is above its isoelectric point ( $\mathrm{pI}$ 4.6), resulting in a negative charge. As a consequence, its association with the positively charged CS/CRG nanoparticles is favored, causing a decrease in the zeta potential value.

As it can be observed in Figure 2, protein entrapment ranged between 12 and 18\% [Fig. 2(a)] and the loading of ovalbumin varied within 4 and 17\% [Fig. 2(b)]. Incorporating different initial amounts of ovalbumin $(10,20$, and $30 \%)$ to the formulation of CS/ CRG nanoparticles 3.5/1 did not lead to significant differences in the association efficiency [Fig. 2(a)].

However, in the formulation $4 / 1$, there was a statistical significant difference $(p<0.05)$ between the association efficiencies of 10,20 , and $30 \%$; the maximum association observed for the $30 \%$ ovalbumin $(17 \%$ of association) $(p<0.05)$. This is probably the result of adding a higher amount of protein, so that more protein is available for interaction with the chitosan molecules, although the maximum difference is only of $5 \%$ (comparing the $17 \%$ association of the formulation with $30 \%$ of initial amount, with the $12 \%$ association of the formulation with $10 \%$ initial amount). The different behavior observed between the formulations CS/CRG 3.5/1 and 4/1, one revealing concentration dependent association and the other an absence of this effect, could be attributed to the highest amount of carrageenan present in the formulation 3.5/1, which could therefore compete with ovalbumin for the chitosan amino groups.

Comparing the association obtained for the formulation CS/CRG $=3.5 / 1$ with that of $4 / 1$ for each of the concentrations, a significant difference $(p<0.05)$ is found only in the case of $10 \%$ of initial amount of ovalbumin, the formulation $3.5 / 1$ associating $18 \%$ as compared with the $12 \%$ of $4 / 1$. The highest loading
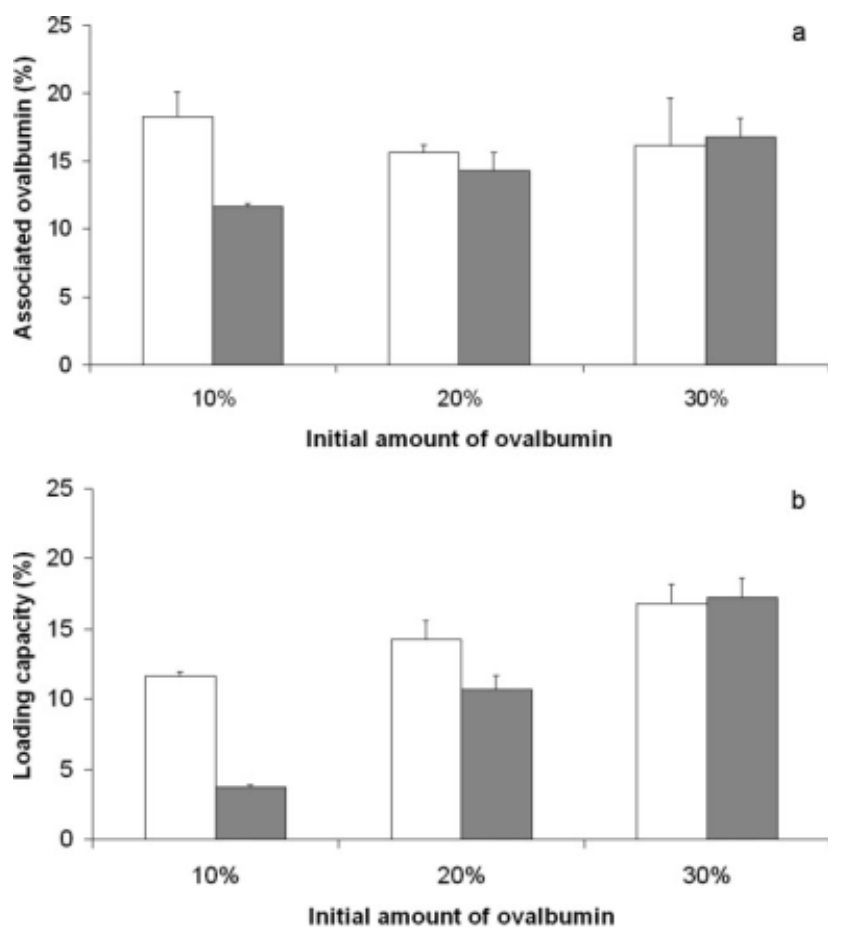

Figure 2. (a) Encapsulation efficiency and (b) loading capacity of ovalbumin in chitosan/carrageenan (CS/CRG) nanoparticles $(\square \mathrm{CS} / \mathrm{CRG}=3.5 / 1 ; \square \mathrm{CS} / \mathrm{CRG}=4 / 1$ ) $(n=3$; mean $\pm \mathrm{SD})$. 


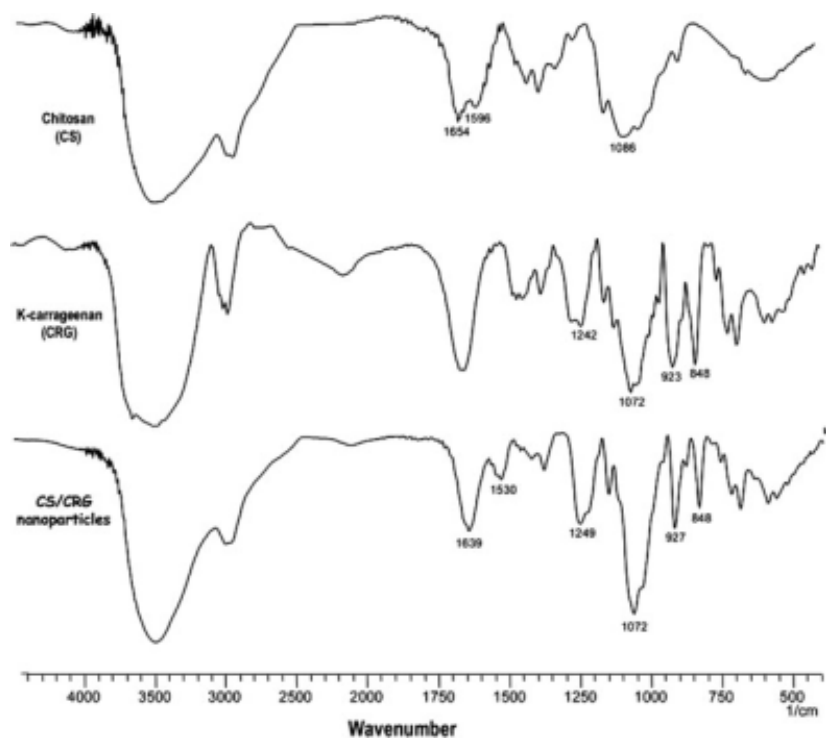

Figure 3. FTIR spectra of chitosan (CS), k-carrageenan (CRG), and CS/CRG nanoparticles.

efficiency (around 17\%) was observed for the formulations to which an initial amount of $30 \%$ ovalbumin was added. This is closely related to the fact that these formulations associated a higher amount of protein, while maintaining a production yield similar to the other formulations, so that they have more protein in the same amount of total solids.

\section{FTIR}

Figure 3 displays the FTIR spectra obtained from the analysis of chitosan, carrageenan, and the nanoparticles obtained by complexation of both polymers. As expected, chitosan revealed the very typical peak of amide I at $1654 \mathrm{~cm}^{-1} 11,20,21$ and a peak at 1596 $\mathrm{cm}^{-1}$, which has been attributed to amide II. ${ }^{20,21}$ Moreover, a peak corresponding to the glycosidic bonds was identified at $1086 \mathrm{~cm}^{-1} .^{20}$

The FTIR analysis of k-carrageenan showed several peaks, from which it is important to highlight a peak at $1242 \mathrm{~cm}^{-1}$ corresponding to the sulfate groups, a peak at $1072 \mathrm{~cm}^{-1}$ attributed to the glycosidic linkage, a peak at $923 \mathrm{~cm}^{-1}$ which corresponds to the 3,6-anhydrogalactose and another at $848 \mathrm{~cm}^{-1}$, corresponding to galactose-4-sulfate. ${ }^{11,22,23}$ The FTIR spectra of the nanoparticles obtained upon complexation of chitosan and carrageenan showed a new absorption band at $\sim 1530 \mathrm{~cm}^{-1}$, assigned to $\mathrm{NH}_{3}^{+}$ groups, which is absent in both spectra of chitosan and carrageenan. Both amide peaks of chitosan converted into a singlet band at $1639 \mathrm{~cm}^{-1}$, and $\mathrm{NH}_{2}$ groups are still identified because not all became protonated and available for interaction with carrageenan. Typical bands of carrageenan were identified in the nanoparticles spectrum, such as sulfate groups at $1249 \mathrm{~cm}^{-1}, 3,6$-anhydrogalactose at 927 $\mathrm{cm}^{-1}$, and galactose-4-sulfate at $848 \mathrm{~cm}^{-1}$. These results evidence the formation of nanoparticles by complexation between the oppositely charged polysaccharides chitosan and carrageenan.

\section{In vitro release studies}

Figure 4 depicts the release profile of ovalbumin from nanoparticles (CS/CRG ratio of 3.5:1) in PBS $\mathrm{pH}$ 7.4 at $37^{\circ} \mathrm{C}$. As can be observed, the developed chitosan/carrageenan nanoparticles showed a sustained release of ovalbumin for a period of 3 weeks. This pattern of release is very promising because it is not only very close to zero order release kinetics, but also because it is sustained for an extended period.

This in vitro release behavior indicates that ovalbumin is in fact encapsulated in the solid matrix of the nanoparticles, not just being distributed through the particles' surface, which usually leads to an initial burst effect. Therefore, it is suggested that ovalbumin established a strong interaction with the nanoparticles. Given the negative charge of ovalbumin ( $\mathrm{pH}$ above its isoelectric point), the protein is able to interact with the positively charged chitosan amino groups. This controlled release profile is extremely important, since it allows incorporating a determined amount of molecule inside a carrier, which will release for a prolonged period of time without the need of repeated administrations. If we consider the use of these nanocarriers, for instance, in the field of tissue engineering/regenerative medicine, the particles could provide an adequate release profile for a specific growth factor on implantation of the device in the affected area.

\section{In vitro cytotoxicity studies}

Figure 5 displays the relative viability of L929 cells upon exposure to different concentrations of CS/

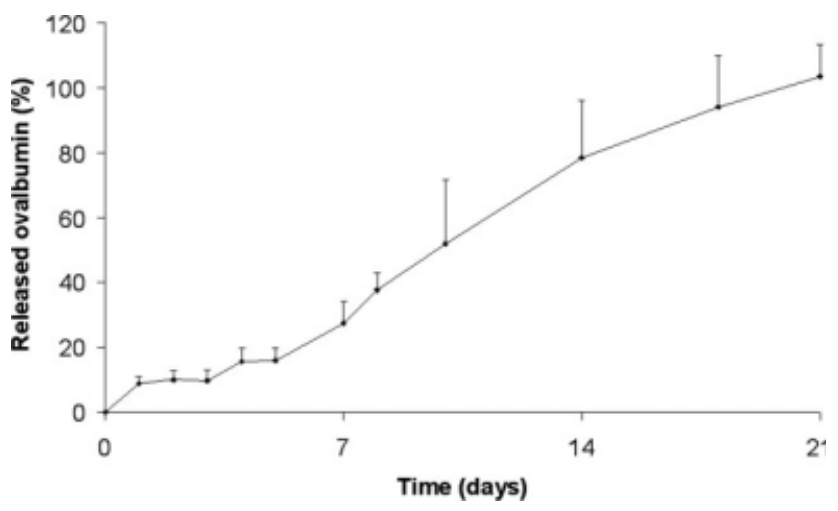

Figure 4. Ovalbumin release profile from chitosan-carrageenan $(\mathrm{CS} / \mathrm{CRG}=3.5 / 1)$ nanoparticles in PBS pH 7.4 at $37^{\circ} \mathrm{C}$ (ovalbumin $=30 \%(\mathrm{w} / \mathrm{w})$ based on chitosan; mean \pm $\mathrm{SD} ; n \geq 3)$. 


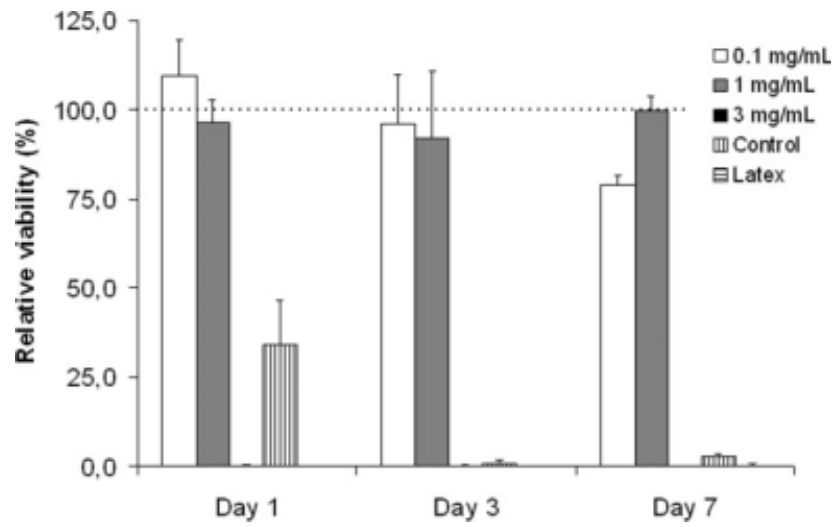

Figure 5. L929 relative cell viability measured by MTS cytotoxicity assay after 1,3 , and 7 days exposure to increasing concentrations of chitosan/carrageenan nanoparticles. Data represent mean \pm SEM $(n=3$ experiments, six replicates per experiment at each test concentration).

CRG nanoparticles (0.1, 1 , and $3 \mathrm{mg}$ nanoparticles/ $\mathrm{mL}$ ) for 1, 3, and 7 days. As can be observed, the lowest concentrations of nanoparticles, 0.1 and $1 \mathrm{mg} / \mathrm{mL}$, generally did not evidence a significant effect on cells viability, when compared with cells incubated with culture medium. The cells show a viability of over $80 \%$ during the experiments with those concentrations of nanoparticles when compared with the negative control.

The highest concentration of nanoparticles assayed evidenced low levels of cellular viability $(p<0.05)$, similar to the latex leachables used as positive control. However, it is important to highlight here that the production of nanoparticles implies their resuspension in water on isolation by centrifugation. This step cannot be avoided and the amount of water was reduced to the minimum possible. Nevertheless, given the high concentration of nanoparticles needed for the assay $(3 \mathrm{mg} / \mathrm{mL})$, the amount of cell culture medium was low and the nutrients provided for the L929 cells are likely to be insufficient to keep the cells growing. Therefore, we believed that the observed cytotoxicity is probably due to the lack of sufficient culture medium (and thus nutrients for the cells) and not to the presence of the nanoparticles itself. To demonstrate this hypothesis, an experimental control was tested by culturing L929 cells in a solution with the same proportion of water/culture medium as used for the $3 \mathrm{mg} / \mathrm{mL}$ nanoparticles concentration. As observed in Figure 5, the obtained relative viability of this control is generally similar to that displayed for the nanoparticles at a concentration of $3 \mathrm{mg} / \mathrm{mL}$. Therefore, in summary, the developed chitosan/carrageenan nanoparticles were demonstrated to be noncytotoxic, under a range of concentrations between 0.1 and $3 \mathrm{mg} / \mathrm{mL}$.

\section{CONCLUSIONS}

This work demonstrates that the newly developed chitosan and carrageenan nanoparticles are suitable to be used as controlled and sustained drug release systems. These nanoparticles are produced in complete hydrophilic conditions, by a very mild procedure of ionic interaction between the positively charged chitosan amino groups and the oppositely charged sulfate groups of carrageenan. This procedure avoids the use of organic solvents and other aggressive conditions that might be detrimental for the integrity of the drug to be released. The macromolecule ovalbumin, used in this study as model protein, was efficiently associated to the developed drug delivery systems, as demonstrated by the physicochemical characterization of the system. The in vitro release assay demonstrated that the chitosan/carrageenan nanoparticles provided a sustained and controlled release of the protein for an extended period of three weeks. Moreover, the developed nanocarriers exhibited low toxicity in contact with fibroblast-like cells, which is an encouraging indicator of their biocompatibility and safety. The controlled release profile and the absence of overt toxicity, make these nanoparticles strong candidates for many biomedical applications, as drug delivery carriers and in the context of tissue engineering strategies.

\section{References}

1. Malafaya P, Silva G, Reis R. Natural-origin polymers as carriers and scaffolds for biomolecules and cell delivery in tissue engineering applications. Adv Drug Deliv Rev 2007;59:207233.

2. Kurita K. Chitin and chitosan: Functional biopolymers from marine crustaceans. Marine Biotechnol 2006;8:203-226.

3. Muzzarelli RAA. Chitin. In: Aspinall GO, editor. The Polysaccharides. Orlando: Academic Press; 1985. p 417-450.

4. Singh DK, Ray AR. Biomedical applications of chitin, chitosan and their derivatives. Rev Macromol Chem Phys C 2000;40:69-83.

5. Dornish M, Hagen A, Hansson E, Peucheur C, Vedier F, Skaugrud O. Safety of Protasan ${ }^{\mathrm{TM}}$ : Ultrapure chitosan salts for biomedical and pharmaceutical use. In: Domard A, Roberts GAF, Varum KM, editors. Advances in Chitin Science. Lyon: Jacques Andre; 1997. p 664-670.

6. Hirano S, Seino H, Akiyama Y, Nonaka I. Biocompatibility of chitosan by oral and intravenous administrations. Polym Mater Sci Eng 1988;59:897-901.

7. Lehr CM, Bouwstra JA, Schacht EH, Junginger HE. In vitro evaluation of mucoadhesive properties of chitosan and some other natural polymers. Int J Pharm 1992;78:43-48.

8. Sankalia MG, Mashru RC, Sankalia JM, Sutariya VB. Stability improvement of alpha-amylase entrapped in kappa-carrageenan beads: Physicochemical characterization and optimization using composite index. Int J Pharm 2006;312:1-14.

9. Bixler HJ. The carrageenan connection IV. British Food J 1994;96:12-17. 
10. Prestwich GD, Marecak DM, Marececk JF, Vercruysse KP, Ziebell MR. Controlled chemical modification of hyaluronic acid: Synthesis, applications, and biodegradation of hydrazide derivatives. J Control Release 1998;53:93-103.

11. Tapia C, Escobar Z, Costa E, Sapag-Hagar J, Valenzuela F, Basualto C, Gai MN, Yazdani-Pedram M. Comparative studies on polyelectrolyte complexes and mixtures of chitosan-alginate and chitosan carrageenan as prolonged diltiazem chorhydrate release systems. Eur J Pharm Biopharm 2004;57:65-75.

12. Piyakulawat P, Praphairaksit N, Chantarasiri N, Muangsin N. Preparation and evaluation of chitosan/carrageenan beads for controlled release of sodium diclofenac. AAPS PharmsciTech 2007;8:E1-E11.

13. Kim K, Fisher JP. Nanoparticle technology in bone tissue engineering. J Drug Target 2007;15:241-252.

14. Silva GA, Ducheyne P, Reis RL. Materials in particulate form for tissue engineering. I. Basic concepts. J Tissue Eng Regener Med 2007;1:4-24.

15. Terbojevich M, Cosani A, Muzzarelli RAA. Molecular parameters of chitosans depolymerised with the aid of papain. Carb Polym 1996;29:63-68.

16. Calvo P, Remuñan-Lopez C, Vila-Jato JL, Alonso MJ. Novel hydrophilic chitosan-polyethylene oxide nanoparticles as protein carriers. J Appl Polym Sci 1997;63:125-132.
17. Sarmento B, Ribeiro A, Veiga F, Ferreira D. Development and characterization of new insulin containing polysaccharide nanoparticles. Colloid Surf B Biointerfaces 2006;53:193202.

18. Fernandez-Urrusuno R, Calvo P, Remuñan-Lopez C, Vila-Jato JL, Alonso MJ. Enhancement of nasal absorption of insulin using chitosan nanoparticles. Pharm Res 1999;16:15761581.

19. Grenha A, Seijo B, Remuñan-Lopez C. Microencapsulated chitosan nanoparticles for lung protein delivery. Eur J Pharm Sci 2005;25:427-437.

20. Schiffman JD, Schauer CL. Cross-linking chitosan nanofibers. Biomacromolecules 2007;8:594-601.

21. Lawrie G, Keen I, Drew B, Chandler-Temple A, Rintoul L, Fredericks P, Grøndahl L. Interactions between alginate and chitosan biopolymers characterized using FTIR and XPS. Biomacromolecules 2007;8:2533-2541.

22. Volery P, Besson R, Schaffer-Lequart C. Characterization of commercial carrageenans by Fourier transform infrared spectroscopy using single-reflection attenuated total reflection. J Agricult Food Chem 2004;52:7457-7463.

23. Abad LV, Relleve LS, Aranilla CT, Rosa AMD. Properties of radiation synthesized PVP-kappa carrageenan hydrogel blends. Rad Phys Chem 2003;68:901-908. 